\title{
A loophole to the universal photon spectrum in electromagnetic cascades: applications to non-thermal primordial nucleosynthesis
}

\author{
P. Serpico* \\ LAPTh - CNRS, Annecy-le-Vieux, France \\ E-mail: serpicodlapth.cnrs.fr \\ V. Poulin \\ LAPTh - CNRS, Annecy-le-Vieux, France \\ E-mail: poulinelapth. cnrs.fr
}

We discuss a loophole of standard electromagnetic cascade theory that can have a large effect in the early universe, notably in altering primordial nucleosynthesis bounds on electromagnetically decaying relic particles. We show how this may greatly simplify the possibility to address the long-standing "lithium problem" in terms of new physics models.

18th International Conference From the Planck Scale to the Electroweak Scale 25-29 May 2015

Ioannina, Greece

${ }^{*}$ Speaker. 


\section{Introduction}

The injection of a energetic photon $(\gamma)$ or electron $(e)$ in a medium filled with radiation, magnetic fields and matter triggers a non-trivial evolution of $\gamma, e^{ \pm}$particle numbers and energy, usually dubbed "electromagnetic cascade". This process is frequently encountered in astroparticle physics, in domains ranging from high-energy gamma-ray astrophysics and ultra-high-energy cosmic ray propagation to the physics of the early universe. The elementary theory of such a cascade onto a photon background has been well known since decades, and can be shown via a textbook derivation (see Chapter VIII in [1], for instance) to lead to a universal "meta-stable" spectrum-attained on timescales much shorter than the thermodynamical equilibration scale—of the form:

$$
\frac{d N_{\gamma}}{d E_{\gamma}}=\left\{\begin{array}{l}
K_{0}\left(\frac{E_{\gamma}}{\varepsilon_{X}}\right)^{-3 / 2} \text { for } E_{\gamma}<\varepsilon_{X}, \\
K_{0}\left(\frac{E_{\gamma}}{\varepsilon_{X}}\right)^{-2} \text { for } \varepsilon_{X} \leq E_{\gamma} \leq \varepsilon_{c} \\
0 \quad \text { for } E>\varepsilon_{c} .
\end{array}\right.
$$

In the above expression, $K_{0}=E_{0} \varepsilon_{X}^{-2}\left[2+\ln \left(\varepsilon_{c} / \varepsilon_{X}\right)\right]^{-1}$ is a normalization constant enforcing that the total energy is equal to the injected electromagnetic energy, $E_{0}$; the characteristic energy $\varepsilon_{c}=$ $m_{e}^{2} / \varepsilon_{\gamma}^{\max }$ denotes the effective threshold for pair-production $\left(\varepsilon_{\gamma}^{\max }\right.$ being the highest energy of the photon background onto which pairs can be effectively created); $\varepsilon_{X} \lesssim \varepsilon_{c} / 3$ is the maximum energy of up-scattered inverse Compton (IC) photons (See [2, 3, 4] for monte carlo studies leading to further justification and parametric evaluations of these quantities).

A notable application of this formalism concerns the possibility of a non-thermal nucleosynthesis phase in the early universe (for reviews on this and other aspects of primordial nucleosynthesis, or BBN, see $[5,6]$ ). The determination of the baryon energy density of the universe $\Omega_{b}$ inferred from the CMB acoustic peaks measurements can be used in fact to turn the standard BBN into a parameter-free theory. The resulting predictions for the deuterium abundance $\left(\right.$ or ${ }^{2} \mathrm{H}$, the most sensitive nuclide to $\Omega_{b}$ ) are in remarkable agreement with observations, providing a tight consistency check for the standard cosmological scenario. The ${ }^{4} \mathrm{He}$ and ${ }^{3} \mathrm{He}$ yields too are, broadly speaking, consistent with this value, and can be used to put constraints on the abundance and lifetime of unstable early universe relics. The ${ }^{7} \mathrm{Li}$ prediction, however, is a factor $\sim 3 \div 4$ above its determination in the atmosphere of metal-poor halo stars, the so-called "lithium problem". If this is interpreted as reflecting a cosmological value — as opposed to a post-primordial astrophysical reprocessing, a question which is far from settled [7, 8] —it requires a non-standard BBN mechanism for which a number of specific possibilities have been explored $[5,6]$. In the last decade, several constraints on non-thermal nucleosynthesis or the determination of specific parameters of exotic scenarios addressing the lithium probelm via cascades rely on the spectrum of Eq. (1.1), see for instance [9].

However, we have recently argued in [10] and [11] that virtually no attention has been paid to the regime when the energy of a photon injected in the primordial plasma, $E_{\gamma}$, falls below the pairproduction threshold. In this case, we found that the universality of the non-thermal spectrum from the standard theory of electromagnetic cascades onto a photon background breaks down. This has major consequences: i) The meta-stable spectrum for such models being non universal, the constraints in the abundance vs. lifetime plane for unstable particles decaying electromagnetically 
in the early universe (derived from ${ }^{2} \mathrm{H},{ }^{4} \mathrm{He}$ and ${ }^{3} \mathrm{He}$ measurements) are non universal as well. In fact, they are often more stringent than commonly thought, up to an order of magnitude. ii) It might yield an unexpected gift, easing the possibility of purely electromagnetic new physics solution to the lithium problem. In the following, we will illustrate these points in some detail.

\section{E.m. cascades, breakdown of universality, and non-thermal BBN}

In general, in order to compute the non-thermal photon spectrum which can photo-disintegrate nuclei, one has to follow the coupled equations of $\gamma$ 's and $e^{ \pm}$populations. If however photons constitute the primary injected particle, it is a good approximation to ignore the non-thermal electrons: while the injected photons will in general Compton scatter and produce them, a further process, typically inverse Compton onto the $\mathrm{CMB}$, is needed to channel back part of their energy in the photon channel. The energy of these photons is significantly lower than the injected one: whenever they are re-injected below nuclear photo-dissociation thresholds they are actually lost for BBN, otherwise they would contribute to strengthening the bounds. Since this does not affect our arguments and is only a technical complication which can be easily dealt with we will neglect these secondary photons. For simplicity, let us also start by assuming that all photon interactions are destructive; i.e. photons are not down-scattered to a (useful) lower energy. Within these approximations, the Boltzmann equation describing the non-thermal photon distribution function $f_{\gamma}$ reads:

$$
\frac{\partial f_{\gamma}\left(E_{\gamma}\right)}{\partial t}=-\Gamma_{\gamma}\left(E_{\gamma}, T(t)\right) f_{\gamma}\left(E_{\gamma}, T(t)\right)+\mathscr{S}\left(E_{\gamma}, t\right)
$$

where $\mathscr{S}\left(E_{\gamma}, t\right)$ is the source injection term, $\Gamma_{\gamma}$ is the total interaction rate, and we neglected the Hubble expansion rate since interaction rates are much faster and rapidly drive $f_{\gamma}$ to a quasi-static equilibrium, $\frac{\partial f_{\gamma}\left(\varepsilon_{\gamma}\right)}{\partial t}=0$. Thus, we simply have :

$$
f_{\gamma}^{\mathrm{S}}\left(E_{\gamma}, t\right)=\frac{\mathscr{S}\left(E_{\gamma}, t\right)}{\Gamma_{\gamma}\left(E_{\gamma}, t\right)},
$$

where the term $\mathscr{S}$ for an exponentially decaying species with lifetime $\tau_{X}$ and density $n_{X}(t)$, whose total e.m. energy injected per particle is $E_{0}$, can be written as

$$
\mathscr{S}\left(E_{\gamma}, t\right)=\frac{n_{\gamma}^{0} \zeta_{X}(1+z(t))^{3} e^{-t / \tau_{X}}}{E_{0} \tau_{X}} p_{\gamma}\left(E_{\gamma}, t\right),
$$

with $z(t)$ being the redshift at time $t$, and the energy parameter $\zeta_{X}$ (conventionally used in the literature) is simply defined in terms of the initial comoving density of the $X$ particle $n_{X}^{0}$ and the actual one of the CMB, $n_{\gamma}^{0}$, via $n_{X}^{0}=n_{\gamma}^{0} \zeta_{X} / E_{0}$. We shall use as reference spectrum the one for a two body decay $X \rightarrow \gamma U$ leading to a single monochromatic line of energy $E_{0}$, corresponding to $p_{\gamma}\left(E_{\gamma}\right)=\delta\left(E_{\gamma}-E_{0}\right)$. If the unspecified particle $U$ is (quasi)massless, like a neutrino, one has $E_{0}=m_{X} / 2$, where $m_{X}$ is the mass of the decaying particle. Note that here, we will be interested in masses $m_{X}$ between a few $\mathrm{MeV}$ and a few hundreds of $\mathrm{MeV}$, and at temperatures of order few $\mathrm{keV}$ or lower, hence the thermal broadening is negligible and a Dirac delta spectrum as the one above is appropriate. We calculate $\Gamma_{\gamma}$ by summing the rates of loss processes, namely:

- Scattering off thermal CMB photons: $\gamma+\gamma_{t h} \rightarrow \gamma+\gamma$, see e.g. [12]. 
- Bethe-Heitler pair creation : $\gamma+N \rightarrow e^{ \pm}+N$, see e.g. [13].

- Compton scattering over thermal electrons: $\gamma+e_{t h}^{ \pm} \rightarrow \gamma+e^{ \pm}$, see e.g. in [3].

Since the critical energy for pair-production is a dynamical quantity, that increases at later times due to the cooling of the universe, it may happen that the primary $\gamma$ energy $E_{0}$ is above threshold for pair-production at early times, and below it at late times (we do take into account that the decay is not instantaneous). In general, at each time we will compare $E_{0}$ with $\varepsilon_{c}$, and use the universal spectrum when $E_{0}>\varepsilon_{c}$, and the solution of Eq. (2.2) when $E_{0}<\varepsilon_{c}$.

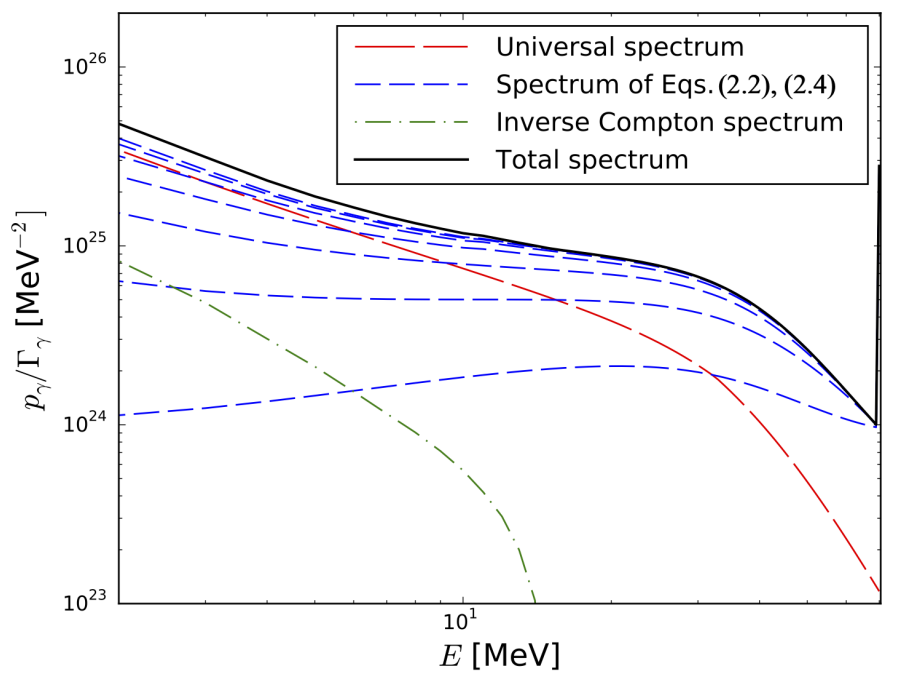

Figure 1: Photon spectrum according to our calculation (solid black line) compared with approximated one used in the literature (long-dashed red), for the case $E_{0}=70 \mathrm{MeV}$ at $T=100 \mathrm{eV}$. The short-dashed, blue lines show the contribution from the $\gamma$ population as computed in our iterative treatment, with the number of iterations increasing from 1 to 7 from bottom to top. The dot-dashed curve is the estimated contribution to the $\gamma$ spectrum from the non-thermal population of $e^{ \pm}$excited by the energy loss channels of the $\gamma^{\prime}$ s.

In reality, not all scattered photons will be "lost": even ignoring the energy transferred to $e^{-}$ and $e^{+}$, Compton scattering and $\gamma \gamma$ scattering still leave lower-energy photons in the final state. This effect can be accounted for by replacing the rhs of Eq. (2.1) by the following term:

$$
\mathscr{S}\left(E_{\gamma}, t\right) \rightarrow \mathscr{S}\left(E_{\gamma}, t\right)+\int_{E_{\gamma}}^{\infty} d x K_{\gamma}\left(E_{\gamma}, x, t\right) f_{\gamma}(x, t) .
$$

The additional term of which the kernel is $K$ accounts for scattered photons and is obtained by summing the differential rates for the $\gamma \gamma$ scattering off background photons and the Compton scattering over thermal electrons, that can be found e.g. in [12]. The integral in Eq. (2.4) now depends on $f_{\gamma}$. We numerically solve this Boltzmann equation using an iterative method: we start from the Dirac distribution and the algebraic solution of Eqs. (2.2) and (2.3), plug in the result thus obtained in Eq. (2.4) to estimate the new "effective" source term, and proceed. Note that the zeroth-order solution of Eqs. (2.2) and (2.3) is exact at the end point $E_{\gamma}=E_{0}$, with further iterations essentially improving the description at lower and lower energies. We stop iterating when the resulting improvement on the constraints is smaller than $3 \%$. Figure 1 shows the resulting spectrum 
proportional to $f_{\gamma}$ according to the prefactor of Eq. (2.3) for an injected monochromatic photon of $70 \mathrm{MeV}$ at the temperature $T=100 \mathrm{eV}$ in the commonly used universal spectrum approximation (long-dashed red line) and for the actual solution of the Boltzmann equation, as a function of the iteration (short-dashed blue lines). For this example, one can estimate $\varepsilon_{c} \simeq 100 \mathrm{MeV}$ and $\varepsilon_{X} \simeq 30 \mathrm{MeV}$. Two features are clearly visible: i) the universal spectrum grossly fails for $E_{\gamma} \gtrsim \varepsilon_{X}$, as expected, since it imposes an artificial suppression; ii) the exact solution is significantly harder at intermediate energies, but attains the same slope as the universal spectrum at low energies (albeit in general with a different low-energy normalization).

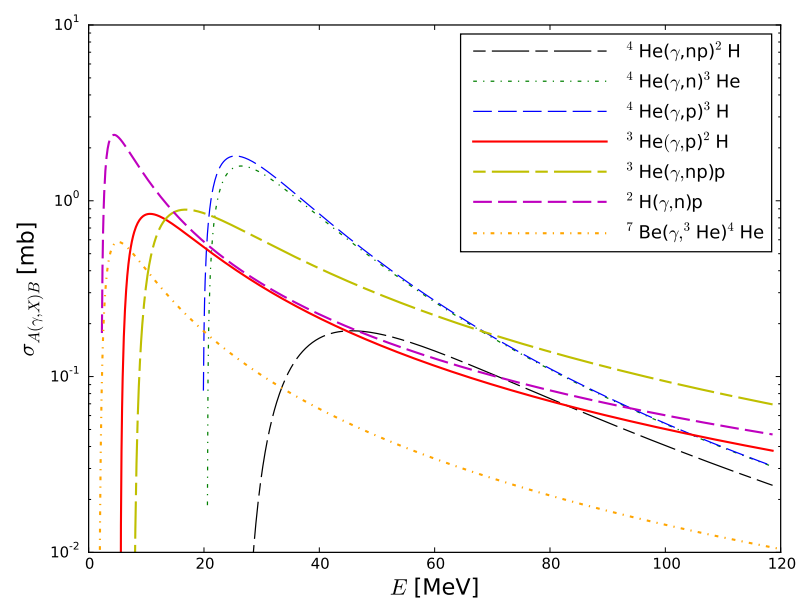

Figure 2: Cross sections for the relevant photodisintegration processes.

At temperatures of few $\mathrm{keV}$ or lower, the standard BBN is over, and the additional nucleosynthesis can be simply dealt with as a post-processing of the abundances computed in the standard scenario, for which we use the input values from Parthenope [15], with the updated value of $\Omega_{b}$ coming from [16]. The non-thermal nucleosynthesis due to electromagnetic cascades can be described by a system of coupled differential equations of the type

$$
\frac{d Y_{A}}{d t}=\sum_{T} Y_{T} \int_{0}^{\infty} d E_{\gamma} f_{\gamma}\left(E_{\gamma}, t\right) \sigma_{\gamma+T \rightarrow A}\left(E_{\gamma}\right)-Y_{A} \sum_{P} \int_{0}^{\infty} d E_{\gamma} f_{\gamma}\left(E_{\gamma}, t\right) \sigma_{\gamma+A \rightarrow P}\left(E_{\gamma}\right)
$$

where: $Y_{A} \equiv n_{A} / n_{b}$ is the ratio of the number density of the nucleus $A$ to the total baryon number density $n_{b}$ (this factors out the trivial evolution due to the expansion of the universe); $\sigma_{\gamma+T \rightarrow A}$ is the photodissociation cross section of the nucleus $T$ into the nucleus $A$, i.e. the production channel for $A ; \sigma_{\gamma+A \rightarrow P}$ is the analogous destruction channel. Both cross sections are actually vanishing below the corresponding thresholds. The set of Eqs. 2.5 is usually solved with respect to redshift $z$, via the transformations reported in [10] and [11]. In general one also needs to follow secondary reactions of the nuclear byproducts of the photodissociation, which can spallate on or fuse with background thermalized target nuclei but none of that is relevant for the problem at hand (see [10] and [11] and refs. therein for details). 


\section{Constraints from the CMB}

A late injection of photons in the thermal bath can lead to additional measurable cosmological alterations. For instance, the injection of a significant amount of energy can lead to modification of the photon-baryon ratio $\eta$ or equivalently, to the increase of the co-moving entropy. Since the inferred values of $\Omega_{b}$ at BBN and CMB epoch are compatible, no major entropy release could have taken place between the two epochs. For a radiation-dominated Universe and by for instantaneous decay at $t \sim \tau$, we have for a small fractional change in entropy (see e.g. [17]):

$$
\frac{\Delta S}{S} \simeq \ln \frac{S_{f}}{S_{i}}=2.14 \times 10^{-4}\left(\frac{\zeta_{X \rightarrow e . m .}}{10^{-9} \mathrm{GeV}}\right)\left(\frac{\tau_{\mathrm{x}}}{10^{6} \mathrm{~s}}\right)^{1 / 2}
$$

where $\zeta_{X \rightarrow \gamma}$ has been traded for $\zeta_{X \rightarrow \text { e.m. }}$. since any electromagnetically interacting decay product can modify the photon-baryon ratio. The $2-\sigma$ limit around the measured value of $\Omega_{b} h^{2}$ by Planck translates into a constraint on the entropy variation of 0.022 [16].

Furthermore, as reviewed in detail in [18], the spectrum of the CMB itself can also be affected through two types of distortion: a modification of the effective chemical potential $\mu$ and a modification of the Compton- $y$ parameter, which is related to the energy gained by a photon after a Compton scattering. To first order, it is possible to distinguish a " $\mu$-distortion epoch" from a " $y$-distortion epoch", because the rate of the processes which are responsible for one type of distortions dominates at very different time. For the relatively early time we focus on, the constraints come essentially from $\mu$-type distortions. We follow here the results of Ref. [18], which contains improvements with respect to the ones given in [19], notably for $z \lesssim 2 \times 10^{6}$, while [19] is accurate enough at late times (see Fig. 16 in [18]). We used the limit given by COBE on the chemical potential: $|\mu| \leq 9 \times 10^{-5}$ [20]. Further details can be found in our publications [10] and [11].

\section{Applications}

\subsection{Non universal bounds on electromagnetically decaying particles}

We first consider the impact of the new meta-stable spectrum derived above on the constraints on electromagnetically decaying relic particles in the abundance $\left(\zeta_{x}\right)$ vs lifetime $\left(\tau_{x}\right)$ plane.

The most important nuclei for what follows are : ${ }^{4} \mathrm{He}$, which can only be destroyed by non-thermal BBN, hence we just impose the 2- $\sigma$ lower limit on its mass abundance $Y_{p} \equiv 4 n_{{ }^{4} \mathrm{He}} / n_{\mathrm{H}}>0.2368$ from [21]; ${ }^{2} \mathrm{H}$, for which we adopt the $2-\sigma$ limit $2.56 \times 10^{-5}<{ }^{2} \mathrm{H} / \mathrm{H}<3.48 \times 10^{-5}$ from [22]; similar results would follow by adopting the combination value compiled in [5], namely $2.45 \times$ $10^{-5}<{ }^{2} \mathrm{H} / \mathrm{H}<3.31 \times 10^{-5}$, which is also closer to the results of [23]; ${ }^{3} \mathrm{He}$, for which we impose no observational lower limit, but the $2-\sigma$ upper limit from $[24]^{3} \mathrm{He} / \mathrm{H}<1.5 \times 10^{-5}$. For the current application, the relevant network of reactions is reported in Fig. 2 and follows the parameterization in the appendix of [9]. The ${ }^{7} \mathrm{Be}\left(\gamma,{ }^{3} \mathrm{He}\right)^{4} \mathrm{He}$ is not included for the application reported in this section, but will be used in the next section, since it is the process at play when dealing with the cosmological lithium problem $\left({ }^{7} \mathrm{Be}\right.$ is in fact the progenitor of the quasi-totality of ${ }^{7} \mathrm{Li}$ for currently acceptable values of $\eta$ ).

A peculiar features for $\gamma$ 's injected below the pair production threshold is that the final outcome reflects the relative energy distribution of the injected $\gamma$ 's (spectral non-universality). This reflects 

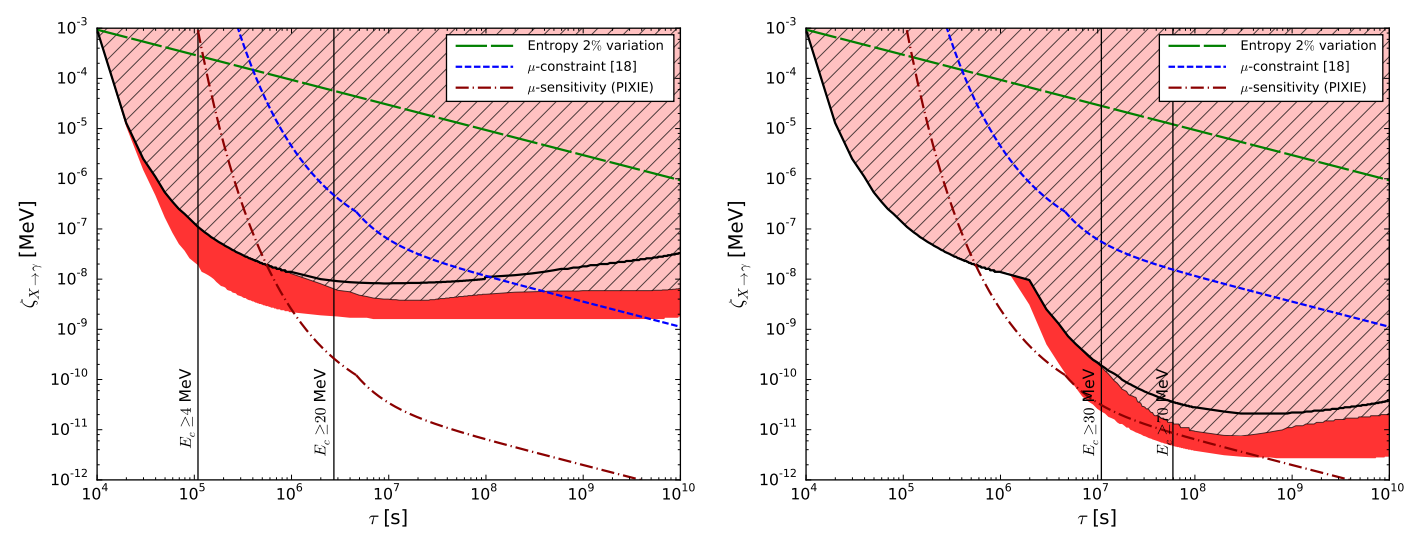

Figure 3: Left Panel - Constraints from ${ }^{2} \mathrm{H}$ depletion in the standard case, with $E_{0} \lesssim 20 \mathrm{MeV}$ (black solid line) and for a non-universal spectrum with $E_{0}=4 \mathrm{MeV}$ (dark shaded red) and $E_{0}=20 \mathrm{MeV}$ (hatched/light shaded red). We also show the sensitivity to entropy variation constraint (green dashed line), current constraints from CMB spectral distortions (excluded above the short-dashed blue line) and the sensitivity reach of the future mission PIXIE [25] (above the red dot-dashed line). Right Panel - Global BBN best constraints in the standard case (black line) and for a non-universal spectrum with $E_{0}=30 \mathrm{MeV}$ (dark shaded red) and $E_{0}=70 \mathrm{MeV}$ (hatched/light shaded red). Right Panel - Global constraints/sensitivities, with the same legend.

the shape of the photo-disintegration cross section, which presents a peak soon after the threshold, followed by a slowly declining tail (see Fig.2). This is why in [11] we chose to illustrate, for each nuclide, the results for two representative examples of monochromatic injection: one close to the resonant peak, and another one well after it. The markedly different outcomes obtained in the two cases prove that constraints depend not only on the decay time and the overall energy injected, but also on the actual energy distribution of the injected photons. Here we report the illustrative case of ${ }^{2} \mathrm{H}$ and the results of the global analysis, addressing the reader to [11] for a more complete treatment. By looking at the reaction network, one can see two regimes appear: at very low energy, below $E_{\gamma} \sim 20 \mathrm{MeV}$, only the destruction of ${ }^{2} \mathrm{H}$ is open (neglecting $A>4$ elements) and constraints have to be derived from this process only. The results in that case are reported in Fig. 3, left panel. At higher energy, all the network of reactions considered is open and the strongest constraints come from ${ }^{2} \mathrm{H}$ depletion at low injection lifetime $\left(\tau_{X} \lesssim 2 \times 10^{6}\right.$ s) and ${ }^{3} \mathrm{He}$ overproduction at higher injection lifetime (right panel). Notice the importance of the non-universality: the two regimes with $30 \mathrm{MeV}$ or $70 \mathrm{MeV}$, and $3 \mathrm{MeV}$ or $20 \mathrm{MeV}$ monochromatic injections lead to significantly different constraints, and in all cases departing from the "universal spectrum" ones.

\subsection{A simple solution to the "lithium problem".}

If the injected energy is $1.59<E_{0} / \mathrm{MeV}<2.22$, the only open non-thermal $\mathrm{BBN}$ channel is ${ }^{7} \mathrm{Be}\left(\gamma,{ }^{3} \mathrm{He}\right){ }^{4} \mathrm{He}$, there are no relevant source terms and only one evolving species (since $Y_{7} \ll Y_{3,4}$ ). Furthermore, the relevant energy range being very small, almost all scattered photons are already below the ${ }^{7} \mathrm{Be}$ photodissociation threshold. In this special case, it is not necessary to use the iterative method described above to get the meta stable spectrum: A monochromatic emission line would simply correspond to $p_{\gamma}\left(E_{\gamma}\right)=\delta\left(E_{\gamma}-E_{0}\right)$ and the final abundance can be expressed as a simple one-dimensional integral (see [10]). In Fig. 4 left panel, the lower band shows for each $\tau_{X}$ 
the range of $\zeta_{X}$ corresponding to a depletion from $40 \%$ to $70 \%$, for the monochromatic photon injection case with $E_{0}=2 \mathrm{MeV}$. Similar results would follow by varying $E_{0}$ by $10 \%$ about this value, i.e. provided one is not too close to the reaction threshold. The upper band represents the analogous region if we had distributed the same injected energy according to the spectrum of Eq. (1.1), up to $\min \left[\varepsilon_{c}, E_{0}\right]$. It is clear that in the correct treatment a large portion of this region survives other cosmological constraints, described below, while none survives in the incorrect treatment.
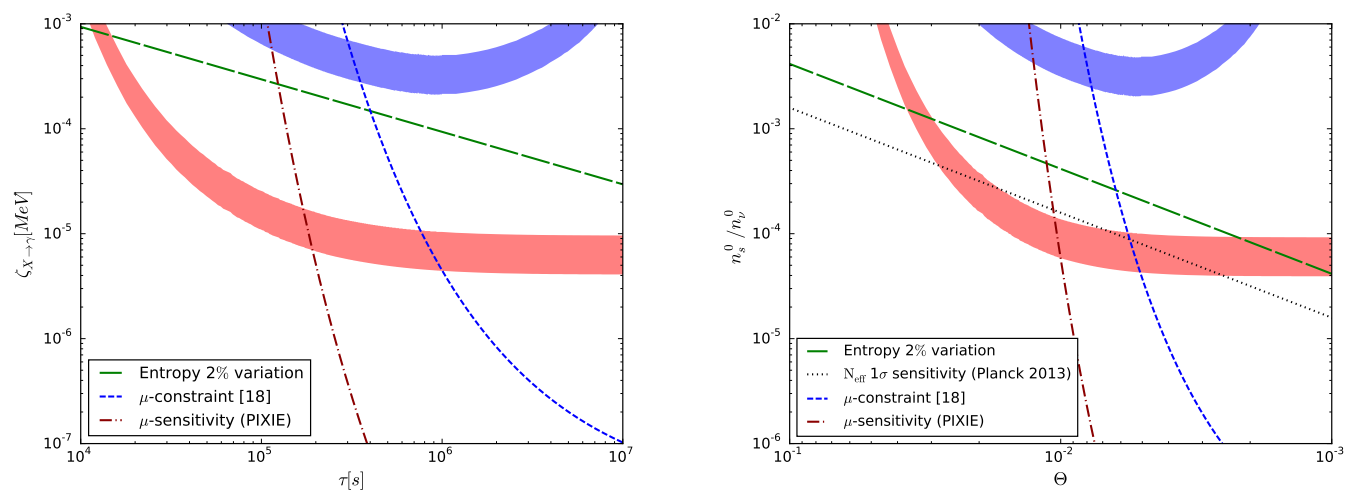

Figure 4: Left Panel - The lower band is the range of abundance parameter $\zeta_{X \rightarrow \gamma}$ vs. lifetime $\tau_{X}$, for which the primordial lithium is depleted to $40 \%$ to $70 \%$ of its standard value, for a monochromatic photon injection with energy $E_{0}=2 \mathrm{MeV}$. The upper band represents the analogous region if we had distributed the same injected energy, up to $E_{0}=2 \mathrm{MeV}$, according to the erroneous spectrum of Eq. (1.1). We again show the sensitivity to entropy variation constraint (green dashed line), current constraints from CMB spectral distortions (excluded above the short-dashed blue line) and the sensitivity reach of the future mission PIXIE [25] (above the red dot-dashed line). Right Panel - Constraints for the sterile neutrino model discussed in the text. The black dotted line correspond to the sensitivity of Planck to $N_{\text {eff }}[16]$. For all other curves, the legend is the same as left panel.

How realistic such a situation is in a concrete particle physics model? It is worth showing as a proof-of-principle that viable models realizing the mechanism described here can be actually constructed. Let us take the simplest case of a sterile Majorana neutrino $v_{s}$ with mass in the range $3.2<M_{s} / \mathrm{MeV}<4.4$, mixing with flavour $\alpha$ neutrinos via an angle $\theta_{\alpha}$. We also define $\Theta^{2} \equiv \sum_{\alpha} \theta_{\alpha}^{2}$. The three main decay channels of $v_{s}$ are (see e.g. [26] and refs. therein): $v_{s} \rightarrow 3 v, v_{s} \rightarrow v_{\alpha} e^{+} e^{-}$, $v_{s} \rightarrow v \gamma$, with branching ratios for the masses of interest and $\theta_{e} \ll \Theta$ of the order of $0.9: 0.1: 0.01$, respectively. It is physically more instructive to normalize the abundance of the $v_{s}, n_{s}^{0}$, in terms of one thermalized $v$ (plus $\bar{v}$ ) flavour species, $n_{v}^{0}$. In Fig. 4 right panel, we show the corresponding range of parameters in the $\Theta-n_{s}^{0} / n_{v}^{0}$ plane, for $M_{s}=4.4 \mathrm{MeV}$, for which the ${ }^{7} \mathrm{Li}$ problem is solved, fulfills cosmological constraints and, provided that $\theta_{e} \ll \Theta$, also laboratory ones [27]. The needed abundance could be obtained in scenarios with low reheating temperature [27]. Note that: i) the entropy release bound is now closer to the region of interest, since the decay mode $v_{s} \rightarrow v_{\alpha} e^{+} e^{-}$, which is useless as far as the ${ }^{7} \mathrm{Be}$ dissociation is concerned, dominates the e.m. energy injection. ii) A non-negligible fraction of relativistic "dark radiation" is now injected, mostly via the dominant decay mode $v_{s} \rightarrow 3 v$; hence we added the current $1 \sigma$ sensitivity of Planck to $N_{\text {eff }}$ [16], with $\Delta N_{\text {eff }}$ computed similarly to what done in [14]. iii) The same type of models had been found incapable of providing an acceptable solution, if the universal spectrum is adopted [14]. 


\section{Conclusions}

We have argued that the universality of the photon spectral shape in electromagnetic cascades has sometimes been used in cosmology beyond its regime of applicability. When the energy of the injected photons falls below the pair-production threshold, i.e. approximately when $E_{\gamma} \lesssim m_{e}^{2} /(22 T) \sim 10 T_{\mathrm{keV}}^{-1} \mathrm{MeV}$, the universal form breaks down. This required the numerical solution of the relevant Boltzmann equations, which we attacked by an iterative scheme. The first implication we have developed is a modification of the BBN constraints on early electromagnetically decaying particles. We have shown that, for illustrative cases of monochromatic energy injection at different epochs, these bounds are i) non universal, in contrary with what was commonly thought; ii) often much stronger than the ones presented in the literature (up to an order of magnitude), notably when the injected photon energy falls close to the peak of the photodisintegration cross-section of the relevant nucleus. In fact, the breaking of the non-universality is non-trivial and is essentially controlled by the energy behavior of the cross-sections: in the universal limit, most of the photons lie at relatively low-energies, so that the cross-section behaviour at the resonance just above threshold is what matters the most. In the actual treatment, the photons may be also sensitive to the high-energy tail of the process. Future studies aiming at assessing the nuclear physics uncertainties affecting these types of bounds would benefit from this insight. It cannot be excluded that in some cases constraints weaken a bit with respect to what considered in the literature.

We also compared BBN bounds with constraints coming from CMB spectral distortions. We concluded that BBN limits are improving over current constraints from COBE via the requirement not to underproduce ${ }^{2} \mathrm{H}$ (at low injection lifetime $\tau_{X}$ ), or not to overproduce ${ }^{3} \mathrm{He}$ (at high $\tau_{X}$ ). The bounds from a future CMB spectral probe as PIXIE would not only greatly improve current CMB constraints, but would also reach the level of current constraints from ${ }^{3} \mathrm{He}$ (often improving over them) allowing for an independent consistency check. This is reassuring, since the cosmological reliability of ${ }^{3} \mathrm{He}$ constraints does stand on some astrophysical assumptions. Below $\tau_{X} \sim 5 \times 10^{5} \mathrm{~s}$, however, ${ }^{2} \mathrm{H}$ constraints would probably remain the most stringent ones for a long time to come. Fortunately they are: i) quite robust, relying on the single, well-known cross-section ${ }^{2} \mathrm{H}(\gamma, n) p$; ii) easy to compute, since no coupled network of equations needs to be solved, the problem reducing to the numerical evaluation of a single integral. This is also the region where constraints coming from hadronic decay modes (not revisited here) are quite stringent.

The possibility to find new mechanisms to deplete the standard BBN prediction of lithium abundance in a consistent way is probably the most spectacular consequence of our investigation. In turn, this could stimulate more specific model-building activities. For instance, decays of relatively light new neutral fermionic particles $X$ for which the $v+\gamma$ channel is the only two body SM channel opened-as it is the case for the light gravitinos in supergravity models-constitute a natural class of candidates. Alternatively, one may think of decaying scenarios involving a pair of quasi degenerate mass states $X$ and $Y$, which are potentially much heavier than the $\mathrm{MeV}$ scale. Some of these scenarios may be motivated by other astroparticle or particle physics reasons and certainly deserve further investigation.

A synergy between BBN and CMB is thus going to be necessary for this kind of physics even in the decades to come. Our work suggests that models in the literature that fulfilled the BBN constraints with less than an order of magnitude margin should perhaps be reconsidered. In 
particular, those characterized by soft gamma-ray emissions and/or at relatively late times should have been more prone to incorrect conclusions about their viability. Our study also suggests that actual bounds should be derived via a case-by-case analysis. Finally, we provided further arguments supporting the usefulness of an improved constraint from CMB spectral distortion of the $\mu$ type, since: i) it can help in testing scenarios able to solve the lithium problem; ii) it would not manifest the unexpected "spectral sensitivity" that we have uncovered.

\section{References}

[1] V. S. Berezinsky et al. "Astrophysics of cosmic rays," Amsterdam, Netherlands: North-Holland (1990) $534 \mathrm{p}$

[2] J. R. Ellis, G. B. Gelmini, J. L. Lopez, D. V. Nanopoulos, S. Sarkar, Nucl. Phys. B 373, 399 (1992).

[3] M. Kawasaki and T. Moroi, Astrophys. J. 452 (1995) 506.

[4] R. J. Protheroe, T. Stanev and V. S. Berezinsky, Phys. Rev. D 51 (1995) 4134.

[5] F. Iocco, G. Mangano, G. Miele, O. Pisanti and P. D. Serpico, Phys. Rept. 472, 1 (2009).

[6] M. Pospelov and J. Pradler, Ann. Rev. Nucl. Part. Sci. 60, 539 (2010).

[7] M. Spite, F. Spite and P. Bonifacio, Mem. Soc. Astron. Ital. Suppl. 22, 9 (2012).

[8] F. Iocco, Mem. Soc. Astron. Ital. Suppl. 22, 19 (2012).

[9] R. H. Cyburt, J. R. Ellis, B. D. Fields and K. A. Olive, Phys. Rev. D 67 (2003) 103521.

[10] V. Poulin and P. D. Serpico, Phys. Rev. Lett. 114, no. 9, 091101 (2015).

[11] V. Poulin and P. D. Serpico, Phys. Rev. D 91, no. 10, 103007 (2015).

[12] R. Svensson and A. A. Zdziarski, Astrophys. J. 349 (1990) 415.

[13] K. Jedamzik, Phys. Rev. D 74 (2006) 103509.

[14] H. Ishida, M. Kusakabe and H. Okada, Phys. Rev. D 90, no. 8, 083519 (2014).

[15] O. Pisanti et al., Comput. Phys. Commun. 178, 956 (2008).

[16] P. A. R. Ade et al. [Planck Collaboration], Astron. Astrophys. 571, A15 (2014).

[17] J. L. Feng, A. Rajaraman and F. Takayama, Phys. Rev. D 68 (2003) 063504.

[18] J. Chluba and R. A. Sunyaev, Mon. Not. Roy. Astron. Soc. 419 (2012) 1294.

[19] W. Hu and J. Silk, Phys. Rev. Lett. 70 (1993) 2661.

[20] D. J. Fixsen et al., Astrophys. J. 473, 576 (1996).

[21] E. Aver, K. A. Olive and E. D. Skillman, JCAP 1204 (2012) 004.

[22] K. A. Olive, P. Petitjean, E. Vangioni and J. Silk, Mon. Not. Roy. Astron. Soc. 426 (2012) 1427.

[23] M. Pettini et al., Mon. Not. Roy. Astron. Soc. 391, 1499 (2008).

[24] T. M. Bania, R. T. Rood and D. S. Balser, Nature 415 (2002) 54.

[25] A. Kogut et al., JCAP 1107 (2011) 025.

[26] F. Bezrukov, H. Hettmansperger and M. Lindner, Phys. Rev. D 81 (2010) 085032.

[27] G. Gelmini, E. Osoba, S. Palomares-Ruiz and S. Pascoli, JCAP 0810, 029 (2008). 